\title{
Überlebensvorteil unter Regorafenib bestätigt
}

\author{
Eine Behandlung mit Regorafenib kann das Gesamtüberleben bei meta- \\ stasiertem Kolorektalkarzinom (mCRC) und fortschreitender Erkrankung trotz \\ Standardtherapie verlängern. Dies bestätigt die aktuelle Auswertung einer \\ Phase-III-Studie. In den USA wurde die Substanz nun zugelassen.
}

Grundlage der Zulassung sind die Ergebnisse der Phase-III-Studie CORRECT. In dieser Studie wurden 760 mCRC-Patienten, deren Erkrankung unter Behandlung mit den verfügbaren Standardtherapieoptionen fortgeschritten war, eingeschlossen. Die Patienten erhielten im Verhältnis 2:1 randomisiert zusätzlich zu Best Supportive Care Regorafenib (Stivarga ${ }^{\circledR}$ ) oder Placebo.

Beim primären Endpunkt der Studie, dem Gesamtüberleben, hatte sich schon in der ersten Interimsanalyse ein signifikanter Vorteil zugunsten der Behandlung mit Regorafenib gezeigt. Dieser Vorteil konnte jetzt in der finalen Analyse der Studiendaten bestätigt werden [van Cutsem E et al. ESMO 2012: LBA18, oral presentation]. Das mediane Gesamtüberleben von 5,0 Monaten im Kontrollarm war unter Regorafenib auf 6,4 Monate verlängert (Hazard Ratio [HR] 0,79; $\mathrm{p}=0,0038$ ). Der Überlebensvorteil zeigte sich dabei in allen vorab definierten Subgruppen.

Der Multi-Kinase-Inhibitor Regorafenib hemmt Zielstrukturen verschiedener Tumorsignalwege: Er inhibiert die Neoangiogenese durch Interaktion mit
VEGFR 1, 2 und 3 (Vascular Endothelial Growth Factor Receptor) und hemmt die Zellproliferation über seine Wirkung auf Rezeptoren wie KIT und PDGFR (Platelet-derived Growth Factor Receptor). Auch die Signaltransduktion in der Tumormikroumgebung wird durch Interaktion mit FGFR (Fibroblast Growth Factor Receptor) inhibiert.

„Die Zulassung für Regorafenib bietet neue therapeutische Optionen für Patienten mit metastasiertem CRC, die bereits alle verfügbaren Möglichkeiten ausgeschöpft haben - eine besonders schwierige Situation für Patienten und Ärzte gleichermaßen“, sagte Prüfarzt Heinz-Josef Lenz, Los Angeles, CA/USA. Doris Berger

Nach Informationen von Bayer

Myelofibrose

\section{Erste spezifische Medikation zugelassen}

\begin{abstract}
Der Januskinasen-Inhibitor Ruxolitinib hat im September die europaweite Zulassung für die zielgerichtete Behandlung der Splenomegalie oder anderer Symptome bei primärer Myelofibrose erhalten.
\end{abstract}

Der Myelofibrose liegt eine Fehlfunktion hämatopoetischer Stammzellen zugrunde. Die mediane Überlebenszeit liegt zwischen zwei und elf Jahren. Ruxolitinib (Ja$\mathrm{kavi}^{\circledR}$ ) ist ein selektiver, oraler Inhibitor der beiden Januskinasen JAK1 und JAK2.

In der COMFORT-I-Studie erhielten 309 Patienten mit primärer und sekundärer Myelofibrose randomisiert über 24 Wochen zweimal täglich oral Ruxolitinib (15 oder $20 \mathrm{mg}$ ) bzw. Placebo [Verstovsek S et al. N Engl J Med. 2012;366(9):799807]. Das primäre Studienziel - Reduktion des Milzvolumens um mindestens $35 \%$ nach 24 Wochen - erreichten unter Ruxolitinib 41,9\% der Studienteilnehmer, unter Placebo nur 0,7\%. In der Verumgruppe erreichten zudem $45,9 \%$ eine $50 \%$ ige Verbesserung des Gesamt-Symptomscores im Vergleich zum Ausgangswert, im Placebo-Arm 5,3\%.

Gute Ergebnisse wurden auch in der COMFORT-II-Studie erzielt [Harrison C et al. N Engl J Med. 2012;366(9):787-98].
Hier erhielten 219 Patienten im Verhältnis 2:1 randomisiert entweder Ruxolitinib oder die „beste verfügbare Therapie“ (best available therapy, BAT - meist Hydroxyurea und andere antineoplastische Sub-

\section{Zulassung erteilt}

Der Proteasom-Inhibitor Bortezomib (Velcade ${ }^{\circledR}$ ) wurde Ende September 2012 für die subkutane Anwendung beim multiplen Myelom zugelassen.

Das positive Votum beruht auf Ergebnissen einer Studie, die zeigte, dass die subkutane Gabe besser verträglich und dabei genauso wirksam ist wie die intravenöse Gabe von Bortezomib [Moreau P et al. Lancet Oncol. stanzen, Glukokortikoide). Nach 48 Wochen zeigten $28 \%$ der Patienten unter Ruxolitinib und kein Patient im BAT-Arm eine mindestens 35\%ige Reduktion des Milzvolumens. Die Lebensqualität stieg in der EORTC QLQ-C30-Skala um 9,1 Punkte unter Verum und um 3,4 Punkte bei BAT an.

Die unter Ruxolitinib auftretenden hämatologischen Nebenwirkungen (Anämie, Thrombopenie) führten selten zum Therapieabbruch. Andreas Fischer

Nach Informationen von Novartis Pharma

\section{Bortezomib-Gabe auch subkutan möglich}

2011;12(5):431-40]. Das Gesamtansprechen war in beiden Gruppen gleich ( $42 \%$ nach vier Zyklen und $52 \%$ nach acht Zyklen). Durch die subkutane Anwendung konnte vor allem die Rate der peripheren Neuropathien deutlich gesenkt werden. Zudem ist die subkutane Gabe eine vielversprechende Alternative für Patienten mit schlechtem Venenzugang. Doris Berger

Nach Informationen von Janssen-Cilag 